\section{Military Technical College Kobry El-Kobbah, Cairo, Egypt}

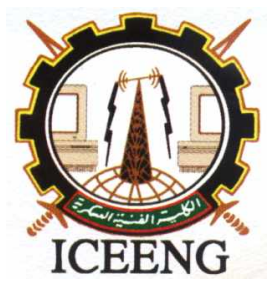

\author{
$6^{\text {th }}$ International Conference \\ on Electrical Engineering \\ ICEENG 2008
}

\title{
Walsh-functions pulse shaping in MSK-type signals.
}

By

\author{
Ibrahim N. Abu-Isbeih*
}

\section{Abstract:}

In this paper a new pulse shaping method in MSK-type signaling is proposed. The proposed pulse shaping method is based on the Walsh functions, and is used to design the optimum pulse shapes in MSK-type signaling. Simulation results show that the proposed pulse shaping method attains improvement in spectral efficiency (i.e., bps/Hz) over MSK, for the same channel bandwidth. The fractional out-of-band power is used to compare the behavior of the system under the new proposed technique. As well, error rate performance in such system is used in the evaluation of this work. The results reveal that good improvement can be attained in spectral efficiency at the expense of error rate performance when these functions are used. The results also reveal that the channel employing MSK system must be as linear as possible for the transmission of such signals.

\section{Keywords:}

Minimum Shift Keying, Walsh Functions, Pulse Shaping.

* Department of Communication Engineering, Philadelphia University, Jordan 


\section{Introduction:}

Several papers have been presented on various modulation schemes. It is very difficult to say which modulation scheme is the best. The selection of a particular optimum modulation technique depends upon the specific system configuration. In most communication system designs, a general objective is to use as efficiently as possible the resources of bandwidth and transmitted power. Thus we are interested in both a modulation scheme's bandwidth efficiency, defined as the ratio of data rate to signal bandwidth (bps/Hz), and its power efficiency, characterized by the error probability which is a function of signal-to-noise ratio (SNR). Minimum Shift Keying (MSK) is considered to be a spectrally efficient modulation scheme, in comparison with other families of constant envelope modulation. However, its main sidelobe can be of some worry in digital data transmission, particularly over nonlinear channels. Therefore, it is practically of interest to search for further efficient pulse shaping schemes, largely proposed either through baseband pulse shaping and/or filtering processing [1,2]. The problem of minimizing out-of-band interference power received a lot of attention in published articles. In the literature [3,4,5], authors have presented analytical and/or numerical techniques for optimizing the baseband pulse shapes of MSK signals. Two discrete pulses have been considered in [4]: the staircase and the piecewise linear, while in $[3,5]$ the optimum pulse is expressed in terms of spheroidal wave functions.

In this paper a new pulse shaping method is proposed based on Walsh functions, where the implementation of Walsh functions is very easy compared to spheroidal wave functions.

\section{Representation of Minimum Shift Keying (MSK):}

The Minimum shift keying (MSK) signal can be defined in the $k$-th interval $k T \leq t \leq(k+1) T$ as [6]:

$x(t)=\cos \left(2 \pi f_{c} t+a_{k} \frac{\pi}{2 T} t+x_{k}\right), k T \leq t \leq(k+1) T$

where $x_{k}$ is a phase constant which is valid over the $k$-th binary data interval. The value of $x_{k}$ is a constant during $T$ interval, that is, $x_{k}=0$ or $\pi$ modulo $2 \pi$, determined by the requirement that the phase of the waveform be continuous at $t=k T$.

Using trigonometric identities and the property that $x_{k}=0, \pi$ (modulo $2 \pi$ ), the MSK waveform representation in (1) can be rewritten in a quadrature form as:

$$
x(t)=a_{I} \cos \left(\frac{\pi t}{2 T}\right) \cos \left(2 \pi f_{c} t\right)+a_{Q} \sin \left(\frac{\pi t}{2 T}\right) \sin \left(2 \pi f_{c} t\right), \quad k T \leq t \leq(k+1) T
$$


where $a_{I}=\cos \left(x_{k}\right)= \pm 1, a_{Q}=-a_{k} \cos \left(x_{k}\right)= \pm 1$ and the in-phase and quadrature symbol weightings are

$$
\begin{aligned}
& c(t)=\cos \left(\frac{\pi t}{2 T}\right), \quad-T \leq t \leq T \\
& s(t)=\sin \left(\frac{\pi t}{2 T}\right), \quad 0 \leq t \leq 2 T
\end{aligned}
$$

As shown by Gronemeyer [6], MSK can be viewed as an Offset-QPSK (OQPSK) with sinusoidal symbol weighting. The in-phase and quadrature channels with the half sinusoidal symbol weighting imposed by $c(t)$ and $s(t)$ respectively. In the case of OQPSK a rectangular symbol weighting used.

From the above discussion and as shown by Gronemeyer [6], we can see that MSK and OQPSK can be generated by demultiplexing the data stream $a_{k}$ into odd and even data streams which are used to determine the symbol pulse signs of the in-phase and quadrature channels during odd intervals: $(2 k-1) T \leq t \leq(2 k+1) T$, and even intervals: $2 k T \leq t \leq(2 k+2) T$, respectively.

MSK can then be viewed as either as an OQPSK signal with sinusoidal pulse weighting, or as a CPFSK signal with modulation index $h=0.5$. The name minimum shift keying comes from the fact that the frequency tone spacing which allows the two FSK signals to be coherently orthogonal is minimum and equals, $\Delta f=1 / 2 T$. The tone spacing in MSK is one-half that employed in conventional orthogonal FSK modulation.

The MSK signal $x(t)$ has a complex (low-pass) envelope of the form:

$\tilde{x}(t)=\exp [j \Phi(t)]=\cos [\Phi(t)]+j \sin [\Phi(t)]$

where

$x(t)=\operatorname{Re}\left[\tilde{x}(t) \exp \left(j 2 \pi f_{c} t\right)\right]$

and $\operatorname{Re}[$.$] denotes the real part of the quantity in brackets. It follows that the baseband$ power spectral density of $x(t)$ is given by [1]:

$$
G(f)=\frac{|C(f)|^{2}+|S(f)|^{2}}{2 T}
$$

where $C(f)$ and $S(f)$ are the Fourier transform of $c(t)$ and $s(t)$ respectively. From (7), the baseband spectrum of MSK signal is then given by: 


$$
G(f)=\frac{16 T}{\pi^{2}}\left[\frac{\cos (2 \pi f T)}{1-16 f^{2} T^{2}}\right]^{2}
$$

A better comparison of bandwidth requirements for these modulation schemes is given in terms of fractional out-of-band power (F.O.B.P), which is given in terms of the baseband power spectrum, $G(f)$, by:

F.O.B.P $=\frac{\int_{W}^{\infty} G(f) d f}{\int_{0}^{\infty} G(f) d f}$

where $W$ is the one-sided bandwidth.

This bandwidth criterion states that the occupied bandwidth is the band that leaves exactly a certain specified percentage outside this bandwidth. For example we will adopt that the bandwidth as the band by which $99 \%$ of the total power is inside the bandwidth (i.e., $1 \%$ of the total power is outside the band). The fractional out-of-band power for MSK is shown in figure (1).

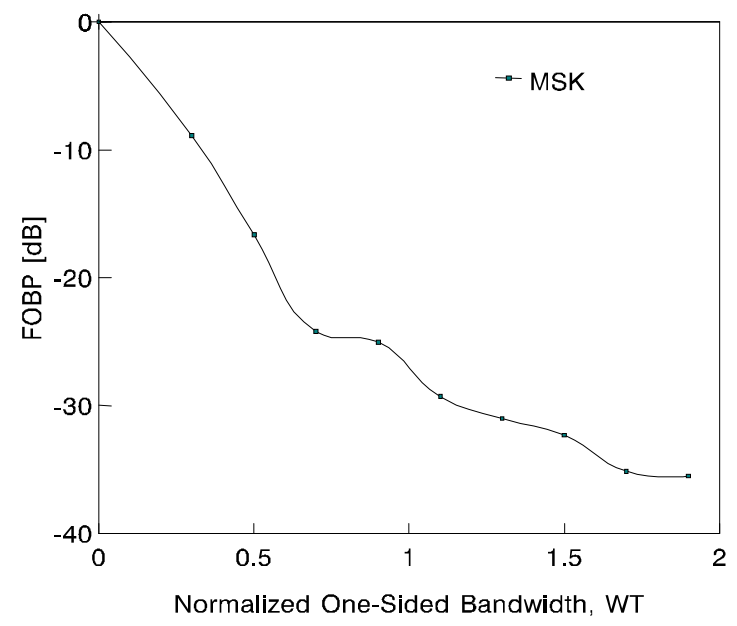

Figure (1): Fractional Out-Of-Band Power for MSK

From figure (1) we can see that the RF bandwidth, $B=2 W$, which contains $99 \%$ of the total power is $B \cong 1.2 R_{b}$ for MSK [1,7].

The bandwidth (spectral) efficiency is defined as $\eta=R_{b} / B$, where $R_{b}=1 / T$ is the bit rate. The bandwidth efficiency for MSK with respect to $99 \%$ power bandwidth can be found as $\eta \cong 0.833 \mathrm{bps} / \mathrm{Hz}$. 


\section{Some Properties of Walsh Functions:}

Before going into details of the pulse shaping techniques in MSK signals, we are going to define the Walsh functions with some of its properties as have been given in [8].

The selection and use of any orthogonal set of functions still hinges primarily on the type of problem under study and the consequent amenability of the problem to the specified set. Walsh function analysis may prove more advantageous from such points of view as manipulation and implementation. Upon defining Walsh functions as a complete orthogonal set, it becomes possible to employ these functions in Walsh series expansion representations for suitable functions. Thus, a square-integrable time-limited function $f(t)$ defined on a finite interval $[0, \mathrm{~T})$ has a Walsh series representation given by:

$$
f(t)=\sum_{n=0}^{\infty} A_{n} \cdot \psi(n, t / T)
$$

where $\psi(n, t / T)$ is the Walsh function and $A_{n}$ is the Walsh coefficient given by

$$
A_{n}=\frac{1}{T} \int_{0}^{T} f(t) \cdot \psi(n, t / T) d t
$$

The proposed pulse shaping in MSK-type signaling is expressed in terms of Walsh functions, as given by (10), this approach is to express the symbol weightings $c(t)$ and $s(t)$ in terms of a finite number $N$ of Walsh functions instead of half sinusoidal pulses. The first eight Walsh functions are shown in figure (2).
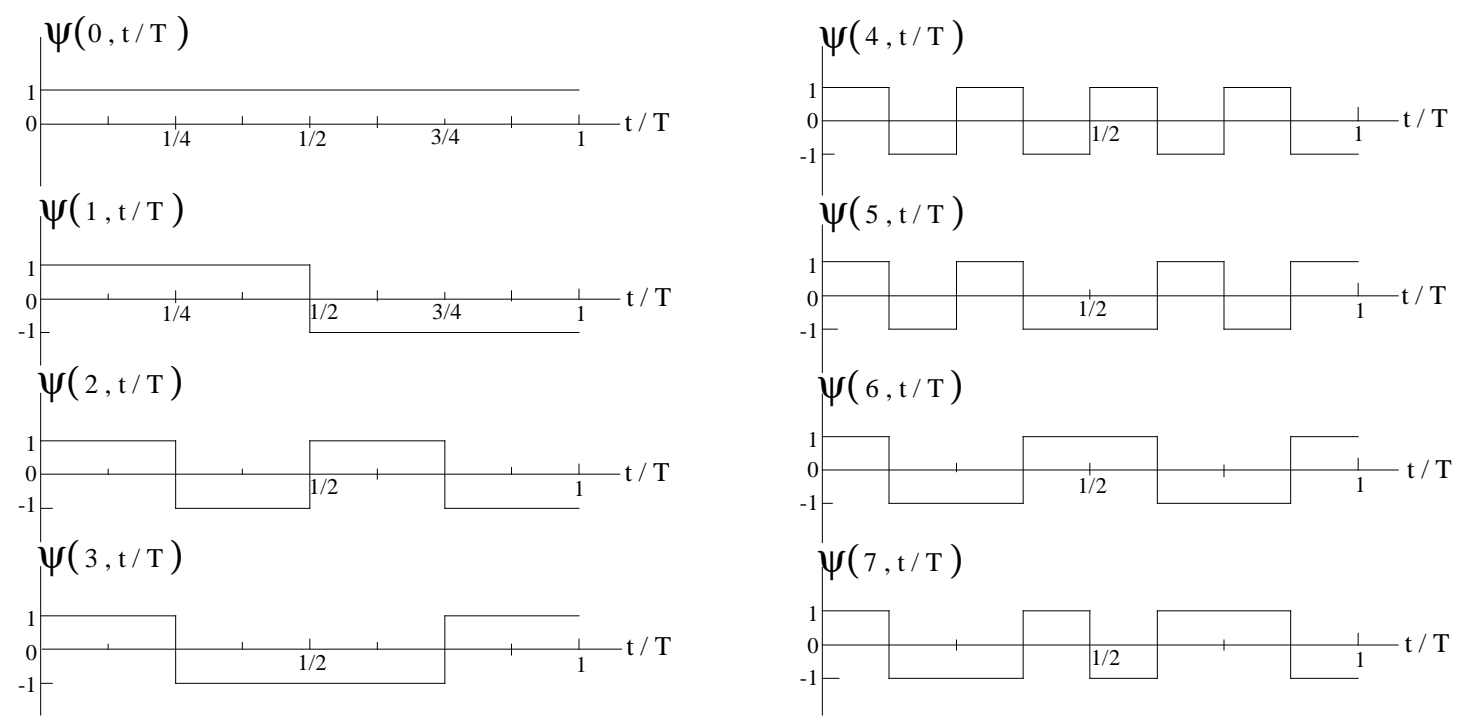

Figure (2): The first eight Walsh functions [8] 


\section{Discrete Symbol Weighting in MSK-Type Signals:}

In MSK the symbol weightings $c(t)$ and $s(t)$ are half-sinusoidal of duration of $2 T$ interval, as have been shown by (3) and (4). In this paper we will use discrete symbol weightings instead of sinusoidal weightings, while keeping with our original idea of generating MSK-type signals. So, we start with representing the new in-phase symbol weighting (pulse) $c(t)$ in terms of Walsh functions on a finite interval $[0, \mathrm{~T})$ as

$$
c(t)=\sum_{n=0}^{\infty} A_{n} \cdot \psi(n, t / T), \quad 0 \leq t<T
$$

Instead of searching for a time continuous optimum pulse $c(t)$, we will search for a discrete version of it for a finite given number $N$ of Walsh functions as given by:

$c_{d}(t)=\sum_{n=0}^{N-1} A_{n} \cdot \psi(n, t / T), \quad 0 \leq t<T$

This discrete pulse $c_{d}(t)$ is defined on one bit interval due to the Walsh functions definition. As in MSK signal the inphase symbol weighting $c(t)$ has even symmetry about its midpoint in the interval $-T \leq t \leq T$; we shall define the discrete symbol weighting $c_{N}(t)$ in the interval $-T \leq t \leq T$ using the even symmetry property, i.e.,

$c_{N}(t)=\left\{\begin{array}{lr}c_{d}(-t), & -T<t<0 \\ c_{d}(t), & 0 \leq t<T\end{array}\right.$

In keeping with our original idea of generating MSK-type signals we shall specify, as is true in MSK, two major requirements, the constant envelope property which is clearly satisfied by symbol weightings $c(t)$ and $s(t)$ in (3) and (4), i.e.,

$$
c^{2}(t)+s^{2}(t)=1, \quad 0 \leq t \leq T
$$

and the second requirement to ensure MSK-type signaling is that the in-phase and quadrature symbol weightings $c(t)$ and $s(t)$ be identical [9], i.e.,

$$
s(t)=c(t-T)
$$

Applying these two requirements on our discrete in-phase and quadrature weightings, we get 
$c_{N}^{2}(t)+s_{N}^{2}(t)=1, \quad 0 \leq t<T$

and

$s_{N}(t)=c_{N}(t-T)$

Furthermore, it is straightforward to show that, the discrete in-phase symbol weighting $c_{N}(t)$ in (14) can be rewritten as

$$
C_{N}(t)= \begin{cases}\sum_{n=0}^{N-1} B_{n} \cdot \operatorname{rect}\left(\frac{-t-T / 2 N-n T / N}{T / N}\right), & -T \leq t<0 \\ \sum_{n=0}^{N-1} B_{n} \cdot \operatorname{rect}\left(\frac{t-T / 2 N-n T / N}{T / N}\right), & 0 \leq t \leq T\end{cases}
$$

Similarly, by following (18) the discrete quadrature symbol weighting $s_{N}(t)$ can be written as

$S_{N}(t)= \begin{cases}\sum_{n=0}^{N-1} B_{n} \cdot \operatorname{rect}\left(\frac{-t-T / 2 N-T-n T / N}{T / N}\right), & 0 \leq t<T \\ \sum_{n=0}^{N-1} B_{n} \cdot \operatorname{rect}\left(\frac{t-T / 2 N-T-n T / N}{T / N}\right), & T \leq t \leq 2 T\end{cases}$

where

$\operatorname{rect}\left(\frac{t-T / 2 N}{T / N}\right)=\left\{\begin{array}{cc}1, & 0 \leq t<T / N \\ 0, & \text { elsewhere }\end{array}\right.$

The new coefficients $B_{n}$ are linear combination of Walsh coefficients $A_{n}$ and are given by the set of linear equations as follows:

$\overline{\mathbf{B}}=\overline{\mathbf{A}} \cdot \overline{\mathbf{W}_{\mathrm{N}}}$

where the vectors $\overline{\mathbf{B}}$ and $\overline{\mathbf{A}}$ are given by:

$\overline{\mathbf{B}}=\left[\begin{array}{llll}B_{0} & B_{1} & \ldots & \ldots \\ B_{N-1}\end{array}\right]$

$\overline{\mathbf{A}}=\left[\begin{array}{llll}A_{0} & A_{1} & \ldots \ldots & A_{N-1}\end{array}\right]$ 
and $\overline{\mathbf{W}_{\mathrm{N}}}$ is an $(N \times N)$ symmetrical matrix with its rows composed of sample values of the first $N$ Walsh functions, e.g., for $N=4$ the matrix $\overline{\mathbf{W}_{4}}$ is given by:

$$
\overline{\mathbf{W}_{4}}=\left[\begin{array}{l}
++++ \\
++-- \\
+-+- \\
+--+
\end{array}\right]
$$

where \pm represent \pm 1 . The discrete in-phase and quadrate symbol weightings $c_{N}(t)$ and $s_{N}(t)$ are shown in figure (3). The matrices $\overline{\mathbf{W}_{2}}, \overline{\mathbf{w}_{4}}, \overline{\mathbf{W}_{8}}$ and $\overline{\mathbf{W}_{16}}$ (for $N=2, N=4, N=8$ and $N=16$ respectively) are shown in [8].
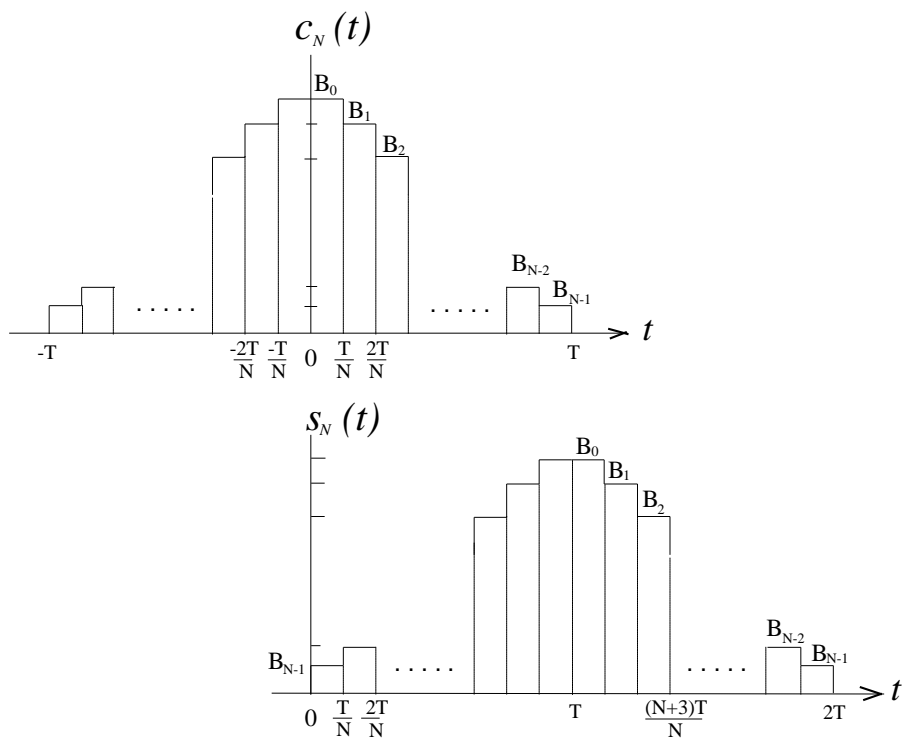

Figure (3): The discrete inphase and quadrature symbol weightings $c_{N}(t)$ and $s_{N}(t)$

To find the baseband power spectral density for the signal $x(t)$, that expressed in (2) with discrete symbol weightings $c_{N}(t)$ and $s_{N}(t)$, we use (7) that is:

$G(f)=\frac{\left|C_{N}(f)\right|^{2}+\left|S_{N}(f)\right|^{2}}{2 T}$

where $C_{N}(f)$ and $S_{N}(f)$ are the Fourier transform of $c_{N}(t)$ and $s_{N}(t)$ respectively; but due to the fact that $c_{N}(t)$ and $s_{N}(t)$ are identical, their Fourier transform magnitudes are equal, i.e.,

$\left|C_{N}(f)\right|=\left|S_{N}(f)\right|$ 
where the baseband power spectral density PSD $G(f)$ in (26) is simplified as follows:

$G(f)=\frac{\left|C_{N}(f)\right|^{2}}{T}$

Then, we are going to search for the optimum pulse shape by finding the coefficients $B_{N}$ which minimize the out-of-band power for a given number of Walsh functions $N$ (the required accuracy) and bandwidth $W$. The fractional out-of-band power (F.O.B.P) is computed numerically from (9). Thus, for each given bandwidth $W$, there exists only one optimum discrete pulse shape. The parameter $C$ has been defined as a function of normalized bandwidth $W T$, and is given by $[4,5]$ :

$$
C=2 \pi W T
$$

The optimization is then carried for different values of bandwidth $W$ (i.e., different values of the parameter $C$ ); for example, $C=1.0$ corresponds to normalized bandwidth $W T=0.159 ; C=3.0$ corresponds to $W T=0.477$; and $C=8.0$ corresponds to $W T=1.273$.

The optimization problem is therefore reduced to the determination of the vector $\overline{\mathbf{B}}$, given by (22), which minimizes (9), subject to the constant envelope property of (17), and is then given by the $N$ following equations:

$B_{i}^{2}+B_{N-1-i}^{2}=1, \quad i=0,1, \ldots ., N-1$

Where, without loss of generality, the constant envelope of the modulated carrier has been set equal to unity. The constraint of constant envelope given by (30) reduces the dimension of the optimization problem in half, as follows:

$B_{i}^{2}+B_{N-1-i}^{2}=1, \quad i=0,1, \ldots \ldots, \frac{N}{2}-1$

where $N$ is even number. In this paper we restrict our work to two cases, the first case with $N=8$ (i.e., the baseband pulses $c_{N}(t)$ and $s_{N}(t)$ are expressed in terms of the first eight Walsh functions), while the other case is for $N=16$ (i.e., the baseband pulses $c_{N}(t)$ and $s_{N}(t)$ are expressed in terms of the first sixteen Walsh functions).

In order to find the PSD, we follow (28), where we will find the Fourier transform of $c_{N}(t)$ and then substitute in (28). For $N=8$ we have the in-phase symbol weighting $c_{N}(t)$ as follows: 


$$
C_{N}(t)= \begin{cases}\sum_{n=0}^{7} B_{n} \cdot \operatorname{rect}\left(\frac{-t-T / 16-n T / 8}{T / 8}\right), & -T \leq t<0 \\ \sum_{n=0}^{7} B_{n} \cdot \operatorname{rect}\left(\frac{t-T / 16-n T / 8}{T / 8}\right), & 0 \leq t \leq T\end{cases}
$$

After some manipulations, the Fourier transform of $c_{N}(t)$ is then given by:

$$
\begin{aligned}
C_{N}(f) & =\int_{-T}^{T} c_{N}(t) e^{-j 2 \pi f t} d t=\frac{2 T \sin \left(\frac{\pi f T}{8}\right)}{\pi f T} \cdot\left\{B_{0} \cos \left(\frac{\pi f T}{8}\right)+B_{1} \cos \left(\frac{3 \pi f T}{8}\right)+B_{2} \cos \left(\frac{5 \pi f T}{8}\right)\right. \\
& \left.+B_{3} \cos \left(\frac{7 \pi f T}{8}\right)+B_{4} \cos \left(\frac{9 \pi f T}{8}\right)+B_{5} \cos \left(\frac{11 \pi f T}{8}\right)+B_{6} \cos \left(\frac{13 \pi f T}{8}\right)+B_{7} \cos \left(\frac{15 \pi f T}{8}\right)\right\}
\end{aligned}
$$

where the baseband PSD of $x(t)$, given by (28), is then:

$$
\begin{aligned}
G(f)= & \frac{4 T \sin ^{2}\left(\frac{\pi f T}{8}\right)}{(\pi f T)^{2}} \cdot\left\{B_{0} \cos \left(\frac{\pi f T}{8}\right)+B_{1} \cos \left(\frac{3 \pi f T}{8}\right)+B_{2} \cos \left(\frac{5 \pi f T}{8}\right)+B_{3} \cos \left(\frac{7 \pi f T}{8}\right)\right. \\
& \left.+B_{4} \cos \left(\frac{9 \pi f T}{8}\right)+B_{5} \cos \left(\frac{11 \pi f T}{8}\right)+B_{6} \cos \left(\frac{13 \pi f T}{8}\right)+B_{7} \cos \left(\frac{15 \pi f T}{8}\right)\right\}^{2}
\end{aligned}
$$

The approach to find the optimum pulse for a given bandwidth $W$ is then considered. For each given $W$ we have one optimum pulse that minimizes the out-of-band power interference given by (9). Our problem is to find the coefficients $B_{i}$ that minimize outof-band power with PSD given by (34). The optimization is carried out by numerical methods; that is, to find the out-of-band power for each given set of $B_{i}$ 's until we reach the minimum out-of-band power at the required bandwidth $W$ (i.e., given $C=2 \pi W T$ ). After finding the coefficients $B_{i}$, we then find the corresponding Walsh coefficients $A_{i}$ using the set of linear equations given by (22).

For $N=8$ the optimum coefficients $B_{i}$ 's are given in Table (1) (i.e., for each given $C$ or $W T$ there is a set of coefficients $B_{i}$ 's corresponding to the optimum pulse at this bandwidth value $W$ ). While the corresponding Walsh coefficients $A_{i}$ are computed according to (22), and are summarized in Table (2). The fractional out-of-band power curves for these obtained optimum pulses are shown in figure (4). From these curves we can observe that for each given bandwidth $W$, the fractional out-of-band power curve for 
the optimum pulse is minimum at this value of bandwidth $W$. (Note that $W$ denotes the bandwidth while $W T$ denotes the normalized bandwidth to the bit rate).

As an example, for $C=3.0$ (corresponding to the normalized bandwidth $W T=0.477$ ), the fractional out-of-band power curve is minimum at this normalized bandwidth value (i.e., $W T=0.477$ ); and so on for other values of $W T$ s (or corresponding $C$ 's).

Similarly for $\mathrm{N}=16$, the inphase symbol weighting $c_{N}(t)$ can be given as

$$
C_{N}(t)= \begin{cases}\sum_{n=0}^{15} B_{n} \cdot \operatorname{rect}\left(\frac{-t-T / 32-n T / 16}{T / 16}\right), & -T \leq t<0 \\ \sum_{n=0}^{15} B_{n} \cdot \operatorname{rect}\left(\frac{t-T / 32-n T / 16}{T / 16}\right), & 0 \leq t \leq T\end{cases}
$$

where the Fourier transform of $c_{N}(t)$ is then given by

$$
\begin{aligned}
C_{N}(f) & =\frac{2 T \sin \left(\frac{\pi f T}{16}\right)}{\pi f T} \cdot\left\{B_{0} \cos \left(\frac{\pi f T}{16}\right)+B_{1} \cos \left(\frac{3 \pi f T}{16}\right)+B_{2} \cos \left(\frac{5 \pi f T}{16}\right)+B_{3} \cos \left(\frac{7 \pi f T}{16}\right)+\right. \\
& +B_{4} \cos \left(\frac{9 \pi f T}{16}\right)+B_{5} \cos \left(\frac{11 \pi f T}{16}\right)+B_{6} \cos \left(\frac{13 \pi f T}{16}\right)+B_{7} \cos \left(\frac{15 \pi f T}{16}\right)+B_{8} \cos \left(\frac{17 \pi f T}{16}\right)+ \\
& +B_{9} \cos \left(\frac{19 \pi f T}{16}\right)+B_{10} \cos \left(\frac{21 \pi f T}{16}\right)+B_{11} \cos \left(\frac{23 \pi f T}{16}\right)+B_{12} \cos \left(\frac{25 \pi f T}{16}\right)+ \\
& \left.+B_{13} \cos \left(\frac{27 \pi f T}{16}\right)+B_{14} \cos \left(\frac{29 \pi f T}{16}\right)+B_{15} \cos \left(\frac{31 \pi f T}{16}\right)\right\}
\end{aligned}
$$

and the baseband PSD for the signal $x(t)$ is then calculated using (28).

Table (3) and Table (4) summarize the optimum coefficients $B_{i}$ and the corresponding Walsh coefficients $A_{i}$ respectively. The fractional out-of-band power curves for these obtained optimum pulses are shown in figure (5).

Figure (6) shows the fractional out-of-band power curves for the optimum pulse $(C=8.0)$ with MSK. From this figure we can see that we can get an improvement by using Walsh functions in pulse shaping with MSK-type signaling where the signal, modulated by the optimum pulse at $C=8.0$, appears spectrally superior to MSK. 
Table (1): The optimum coefficients $B_{i}$ corresponding to the obtained optimum pulses $(N=8)$ for various values of normalized bandwidth WT

\begin{tabular}{|c|c|c|c|c||}
\hline $\begin{array}{c}\text { Bandwidth factor } \\
C=2 \pi W T\end{array}$ & $\begin{array}{c}\boldsymbol{C = 1 . 0} \\
(\boldsymbol{W} \boldsymbol{T = 0 . 1 5 9})\end{array}$ & $\begin{array}{c}\boldsymbol{C = 3 . 0} \\
(\boldsymbol{W T}=\mathbf{0 . 4 7 7})\end{array}$ & $\begin{array}{c}\boldsymbol{C = 4 . 0} \\
(\boldsymbol{W T = 0 . 6 3 7 )}\end{array}$ & $\begin{array}{c}\boldsymbol{C = 8 . 0} \\
(\boldsymbol{W T = 1 . 2 7 3})\end{array}$ \\
\hline \hline$B_{0}$ & 0.80 & 0.90 & 0.98 & 0.996 \\
\hline$B_{1}$ & 0.75 & 0.88 & 0.96 & 0.973 \\
\hline$B_{2}$ & 0.76 & 0.86 & 0.89 & 0.907 \\
\hline$B_{3}$ & 0.73 & 0.75 & 0.78 & 0.785 \\
\hline$B_{4}$ & 0.63 & 0.64 & 0.62 & 0.619 \\
\hline$B_{5}$ & 0.65 & 0.51 & 0.43 & 0.421 \\
\hline$B_{6}$ & 0.60 & 0.46 & 0.28 & 0.231 \\
\hline$B_{7}$ & 0.53 & 0.42 & 0.15 & 0.089 \\
\hline
\end{tabular}

Table (2): The optimum Walsh coefficients $A_{i}$ corresponding to the obtained optimum pulses $(N=8)$ for various values of normalized bandwidth WT

\begin{tabular}{|c|c|c|c|c|}
\hline $\begin{array}{l}\text { Bandwidth factor } \\
\qquad C=2 \pi W T\end{array}$ & $\begin{array}{c}C=1.0 \\
(W T=0.159)\end{array}$ & $\begin{aligned} C & =3.0 \\
(W T & =0.477)\end{aligned}$ & $\begin{aligned} C & =4.0 \\
(W T & =0.637)\end{aligned}$ & $\begin{array}{c}C=8.0 \\
(W T=1.273)\end{array}$ \\
\hline$A_{0}$ & $7.028 \times 10^{-1}$ & $6.825 \times 10^{-1}$ & $6.405 \times 10^{-1}$ & $6.276 \times 10^{-1}$ \\
\hline$A_{1}$ & $6.963 \times 10^{-2}$ & $1.713 \times 10^{-1}$ & $2.67 \times 10^{-1}$ & $2.876 \times 10^{-1}$ \\
\hline$A_{2}$ & $3.038 \times 10^{-2}$ & $5.35 \times 10^{-2}$ & $1.115 \times 10^{-1}$ & $1.246 \times 10^{-1}$ \\
\hline$A_{3}$ & $-2.875 \times 10^{-3}$ & $-1.225 \times 10^{-2}$ & $-4.5 \times 10^{-2}$ & $-5.538 \times 10^{-2}$ \\
\hline$A_{4}$ & $1.088 \times 10^{-2}$ & $3.625 \times 10^{-2}$ & $5.675 \times 10^{-2}$ & $6.063 \times 10^{-2}$ \\
\hline$A_{5}$ & $-8.75 \times 10^{-4}$ & $-7.5 \times 10^{-3}$ & $-2.075 \times 10^{-2}$ & $-2.438 \times 10^{-2}$ \\
\hline$A_{6}$ & $-1.25 \times 10^{-4}$ & $2.25 \times 10^{-3}$ & $-3.75 \times 10^{-3}$ & $-5.375 \times 10^{-3}$ \\
\hline$A_{7}$ & $-4.875 \times 10^{-3}$ & $-2.1 \times 10^{-2}$ & $-1.825 \times 10^{-2}$ & $-1.938 \times 10^{-2}$ \\
\hline
\end{tabular}

Table (3): The optimum coefficients $B_{i}$ corresponding to the obtained optimum pulses $(N=16)$ for various values of normalized bandwidth WT

\begin{tabular}{||c|c|c|c|c||}
\hline $\begin{array}{c}\text { Bandwidth factor } \\
C=2 \pi W T\end{array}$ & $\begin{array}{c}\boldsymbol{C}=\mathbf{1 . 0} \\
(\boldsymbol{W}=\mathbf{0 . 1 5 9})\end{array}$ & $\begin{array}{c}\boldsymbol{C}=\mathbf{3 . 0} \\
(\boldsymbol{W} \boldsymbol{T}=\mathbf{0 . 4 7 7})\end{array}$ & $\begin{array}{c}\boldsymbol{C}=\mathbf{4 . 0} \\
(\boldsymbol{W} \boldsymbol{T}=\mathbf{0 . 6 3 7})\end{array}$ & $\begin{array}{c}\boldsymbol{C}=\mathbf{8 . 0} \\
(\boldsymbol{W} \boldsymbol{T}=\mathbf{1 . 2 7 3})\end{array}$ \\
\hline \hline$B_{0}$ & 0.76 & 0.951 & 0.99 & 0.998 \\
\hline$B_{1}$ & 0.75 & 0.94 & 0.976 & 0.995 \\
\hline$B_{2}$ & 0.74 & 0.918 & 0.968 & 0.982 \\
\hline
\end{tabular}




\begin{tabular}{||c|c|c|c|c||}
\hline$B_{3}$ & 0.73 & 0.898 & 0.944 & 0.965 \\
\hline$B_{4}$ & 0.729 & 0.876 & 0.915 & 0.935 \\
\hline$B_{5}$ & 0.723 & 0.827 & 0.86 & 0.876 \\
\hline$B_{6}$ & 0.719 & 0.78 & 0.818 & 0.836 \\
\hline$B_{7}$ & 0.71 & 0.72 & 0.729 & 0.77 \\
\hline$B_{8}$ & 0.704 & 0.694 & 0.685 & 0.638 \\
\hline$B_{9}$ & 0.695 & 0.626 & 0.575 & 0.549 \\
\hline$B_{10}$ & 0.691 & 0.562 & 0.51 & 0.482 \\
\hline$B_{11}$ & 0.685 & 0.482 & 0.403 & 0.355 \\
\hline$B_{12}$ & 0.683 & 0.44 & 0.33 & 0.262 \\
\hline$B_{13}$ & 0.673 & 0.397 & 0.251 & 0.189 \\
\hline$B_{14}$ & 0.661 & 0.341 & 0.218 & 0.099 \\
\hline$B_{15}$ & 0.65 & 0.309 & 0.141 & 0.063 \\
\hline
\end{tabular}

Table (4): The optimum Walsh coefficients $A_{i}$ corresponding to the obtained optimum pulses $(N=16)$ for various values of normalized bandwidth WT

\begin{tabular}{|c|c|c|c|c||}
\hline $\begin{array}{c}\text { Bandwidth factor } \\
C=2 \pi W T\end{array}$ & $\begin{array}{c}\boldsymbol{C}=\mathbf{1 . 0} \\
(\boldsymbol{W T = 0 . 1 5 9})\end{array}$ & $\begin{array}{c}\boldsymbol{C}=\mathbf{3 . 0} \\
(\boldsymbol{W T = 0 . 4 7 7})\end{array}$ & $\begin{array}{c}\boldsymbol{C}=\mathbf{4 . 0} \\
\boldsymbol{W T} \boldsymbol{T}=\mathbf{0 . 6 3 7})\end{array}$ & $\begin{array}{c}\boldsymbol{C}=\mathbf{8 . 0} \\
(\boldsymbol{W T = 1 . 2 7 3})\end{array}$ \\
\hline \hline$A_{0}$ & $7.064 \times 10^{-1}$ & $6.726 \times 10^{-1}$ & $6.446 \times 10^{-1}$ & $6.246 \times 10^{-1}$ \\
\hline$A_{1}$ & $2.619 \times 10^{-2}$ & $1.912 \times 10^{-1}$ & $2.554 \times 10^{-1}$ & $2.95 \times 10^{-1}$ \\
\hline$A_{2}$ & $1.294 \times 10^{-2}$ & $8.631 \times 10^{-2}$ & $1.118 \times 10^{-1}$ & $1.209 \times 10^{-1}$ \\
\hline$A_{3}$ & $-5.625 \times 10^{-4}$ & $-2.331 \times 10^{-2}$ & $-4.231 \times 10^{-2}$ & $-5.55 \times 10^{-2}$ \\
\hline$A_{4}$ & $8.188 \times 10^{-3}$ & $4.631 \times 10^{-2}$ & $5.319 \times 10^{-2}$ & $5.563 \times 10^{-2}$ \\
\hline$A_{5}$ & $-3.125 \times 10^{-4}$ & $-1.156 \times 10^{-2}$ & $-1.794 \times 10^{-2}$ & $-2.425 \times 10^{-2}$ \\
\hline$A_{6}$ & $-3.125 \times 10^{-4}$ & $-2.438 \times 10^{-3}$ & $-3.063 \times 10^{-3}$ & $-6.125 \times 10^{-3}$ \\
\hline$A_{7}$ & $2.438 \times 10^{-3}$ & $-1.356 \times 10^{-2}$ & $-1.869 \times 10^{-2}$ & $-1.375 \times 10^{-2}$ \\
\hline$A_{8}$ & $4.438 \times 10^{-3}$ & $2.269 \times 10^{-2}$ & $3.469 \times 10^{-2}$ & $2.938 \times 10^{-2}$ \\
\hline$A_{9}$ & $-6.251 \times 10^{-5}$ & $-5.188 \times 10^{-3}$ & $-1.194 \times 10^{-2}$ & $-1.125 \times 10^{-2}$ \\
\hline$A_{10}$ & $-6.251 \times 10^{-5}$ & $-3.125 \times 10^{-4}$ & $-2.813 \times 10^{-3}$ & $1.25 \times 10^{-4}$ \\
\hline$A_{11}$ & $6.875 \times 10^{-4}$ & $-9.438 \times 10^{-3}$ & $-1.044 \times 10^{-2}$ & $-1.325 \times 10^{-2}$ \\
\hline$A_{12}$ & $-6.25 \times 10^{-5}$ & $-1.312 \times 10^{-3}$ & $-2.437 \times 10^{-3}$ & $-1.375 \times 10^{-3}$ \\
\hline$A_{13}$ & $-3.125 \times 10^{-4}$ & $-1.188 \times 10^{-3}$ & $-3.063 \times 10^{-3}$ & $-1.25 \times 10^{-3}$ \\
\hline$A_{14}$ & $4.375 \times 10^{-4}$ & $1.313 \times 10^{-3}$ & $1.563 \times 10^{-3}$ & $-5.125 \times 10^{-3}$ \\
\hline$A_{15}$ & $-6.25 \times 10^{-5}$ & $1.563 \times 10^{-3}$ & $1.438 \times 10^{-3}$ & $4.25 \times 10^{-3}$ \\
\hline
\end{tabular}




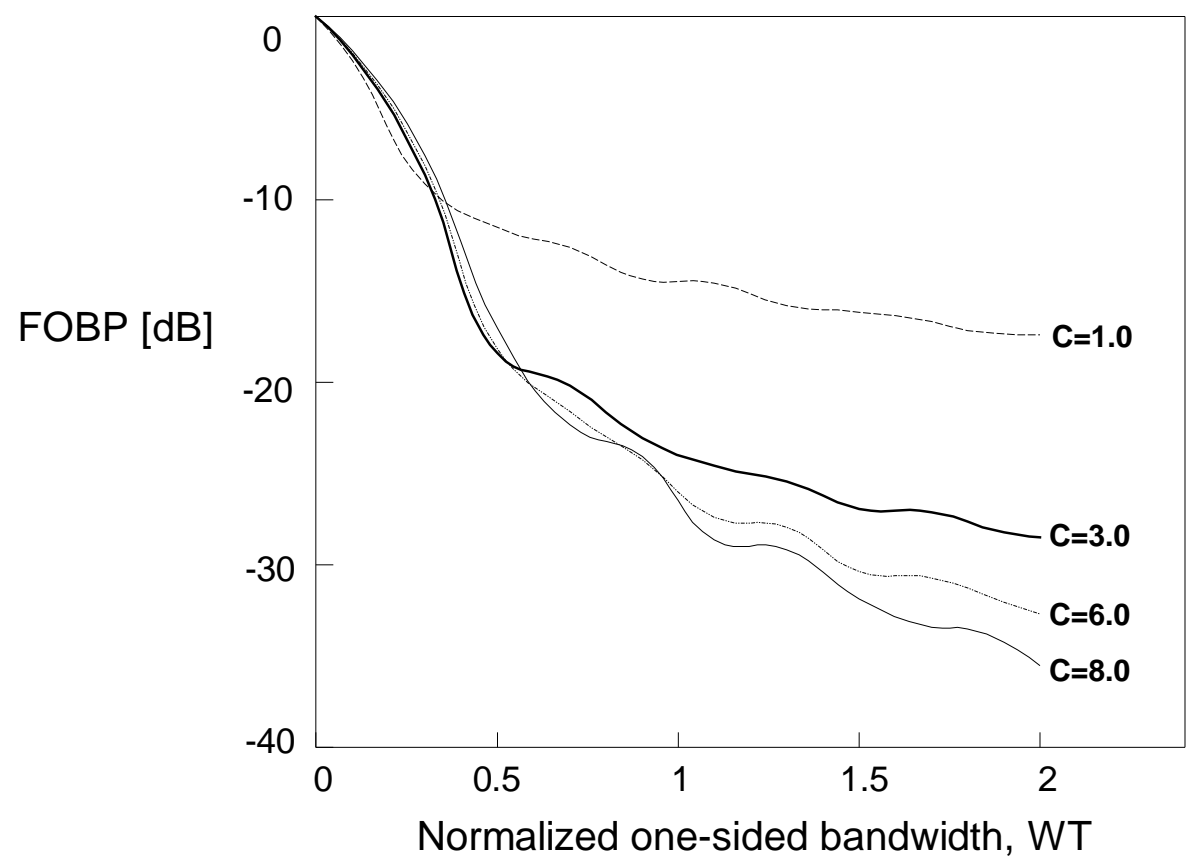

Figure (4): Fractional out-of-band power for optimum pulse shaping with MSK-type signaling $(N=8)$

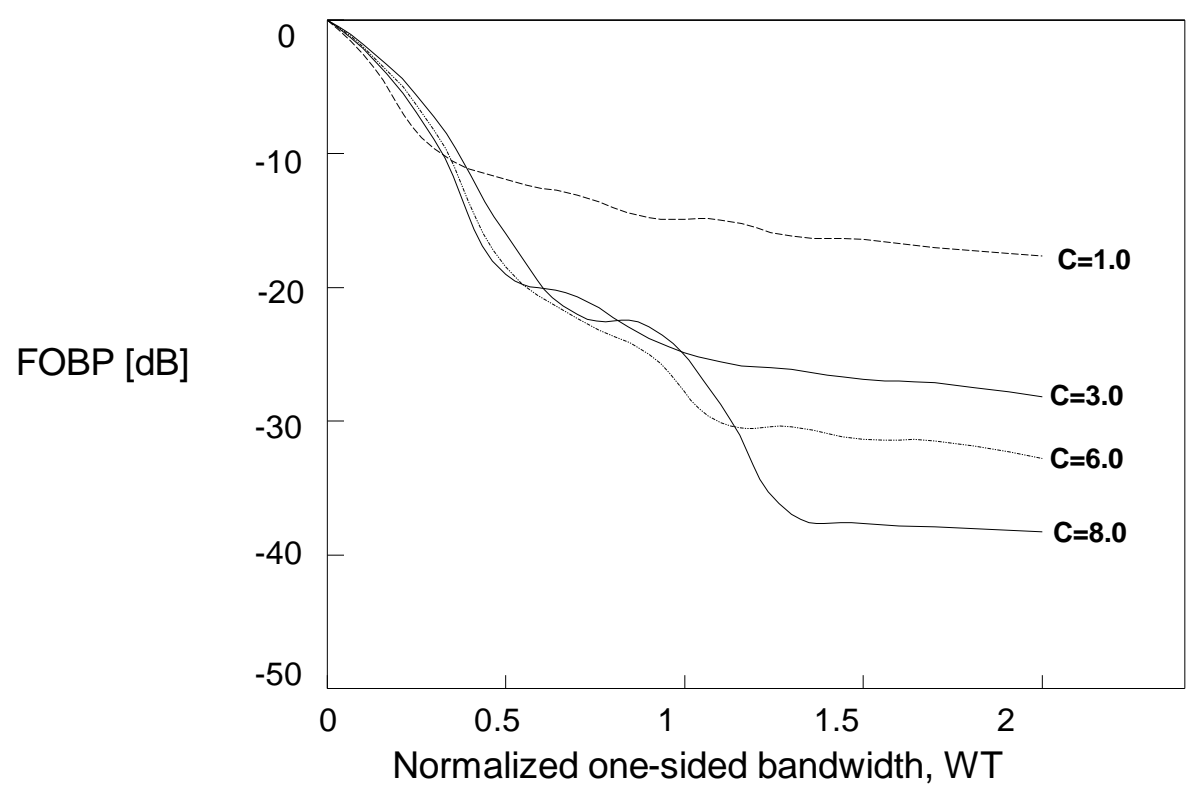

Figure (5): Fractional out-of-band power for optimum pulse shaping with MSK-type signaling $(N=16)$ 


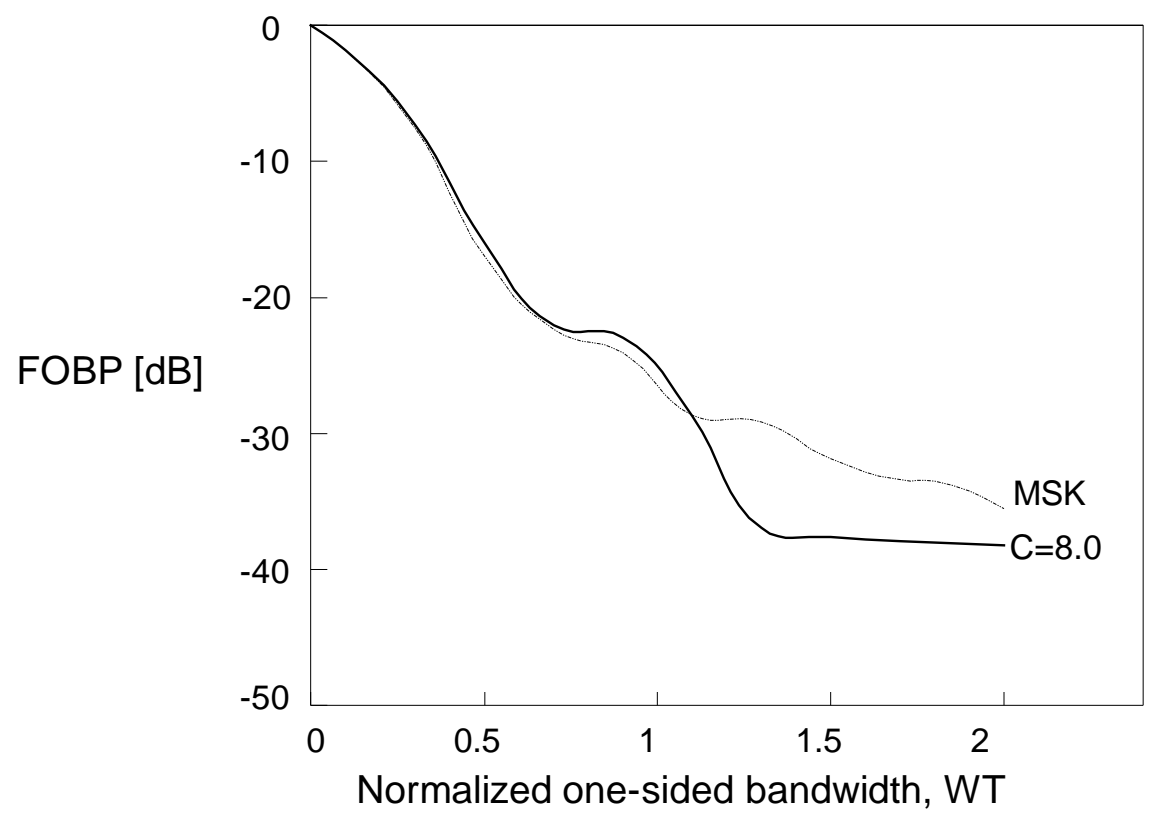

Figure (6): Fractional out-of-band power for optimum pulse shaping $C=8.0(N=16)$, and for MSK

\section{Simulation Results:}

The simulation is carried for the signal modulated by the obtained optimum pulses with $N=16$. The channel models used in this paper are the discrete linear RC-low pass-type channel and the discrete nonlinear Volterra channel with $10 \%$ nonlinear distortion percentage.

The simulation results over a discrete linear channel are shown in Fig. 7, while the simulation results over a nonlinear Volterra channel (10\%) are shown in Fig. 8. From these two figures we can see that the SNR degradation increases as $C \rightarrow 0$ which results from intersymbol interference.

Table (5) summarizes the SNR needed at bit-error rate of $10^{-4}$ for the obtained optimum pulses over linear and nonlinear channels. These results are obtained from figure (7) and figure (8) and compared to the SNR needed for MSK.

From these tables we can see that the optimum pulse under the criterion of out-of-band power may not be the optimum under the criterion of error rate performance. 


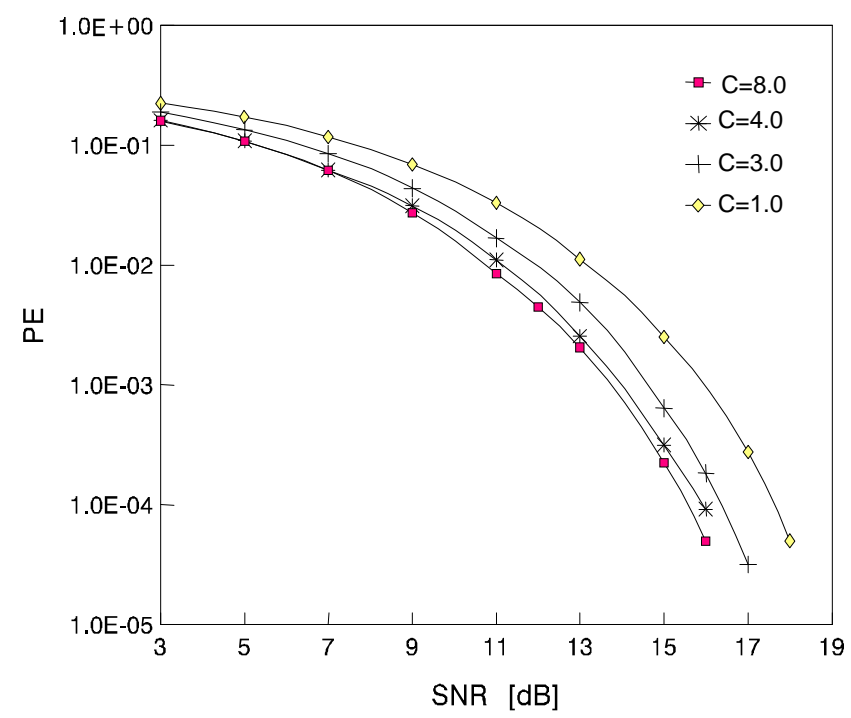

Figure (7): BER for optimum pulses $(N=16)$ for various values of normalized bandwidth WT over a linear channel

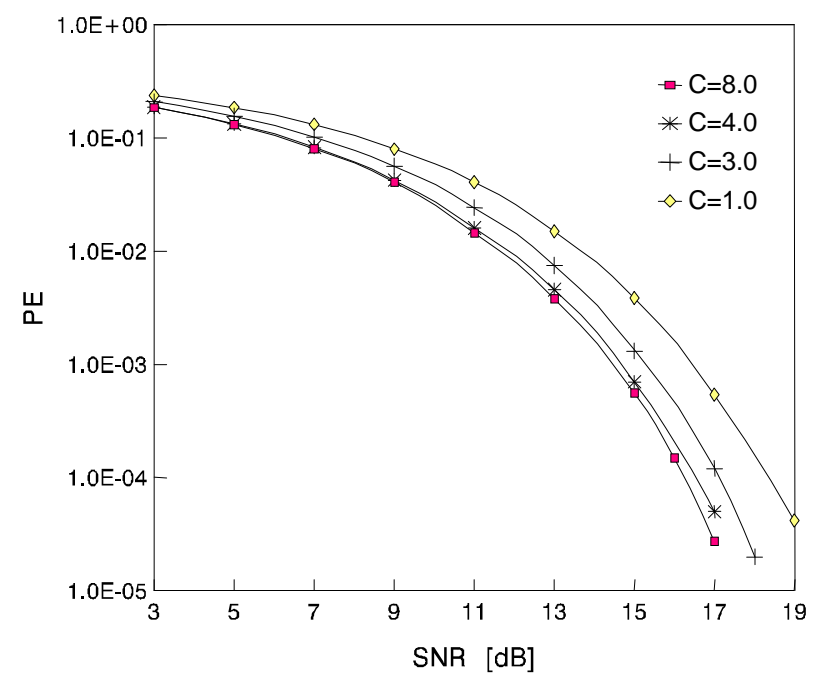

Figure (8): BER for optimum pulses $(N=16)$ for various values of normalized bandwidth WT over a nonlinear Volterra channel (10\%)

Table (5): $S N R(d B)$ needed for the obtained optimum pulses at $P_{e}=10^{-4}$ with $(N=16)$

\begin{tabular}{||c|c|c|c|c|c||}
\hline Modulation Technique & \multirow{2}{*}{ MSK } & \multicolumn{4}{|c|}{ Pulse-Shaped MSK } \\
\cline { 3 - 6 } & & $C=8$ & $C=4$ & $C=3$ & $C=1$ \\
\hline Linear channel & 15.4 & 15.6 & 15.9 & 16.4 & 17.7 \\
\hline Nonlinear channel (10\%) & 16.25 & 16.3 & 16.5 & 17.1 & 18.4 \\
\hline
\end{tabular}




\section{Conclusions:}

In this paper, a new pulse shaping technique is presented based upon Walsh functions. From the above results, we can conclude that the application of Walsh functions with optimum pulse shaping design (i.e., to express the optimum pulse shape in terms of Walsh functions) in MSK-type signaling in order to minimize the fractional out-of-band power leads to the flexibility of using such optimum pulses corresponding to the required bandwidth (channel bandwidth) with minimum fractional out-of-band power. However the criterion should be borne in mind, for the choice which yields highest band occupancy will not usually be best under other desirable criterion, such as error performance; i.e., the obtained optimum pulses may not be optimum under the criterion of error rate performance. The channel used in MSK-type signaling systems must be as linear as possible in order to get acceptable error rate performance.

\section{References:}

[1] R. E. Ziemer and R. L. Peterson, Introduction to Digital Communication, Macmillan Publishing Company, New York, 1992.

[2] D. V. Ramana, A. P. Shivaprasad, and S. Pal, Theoretical and Experimental Results on QPSK Spectrum used for Data Transmission, IEEE TENCON-2003: Proceedings of the Conference on Convergent Technologies for the Asia Pacific, India, October 2003.

[3] G. S. Deshpande and P. H. Wittke, Optimum Pulse Shaping in Digital Angle Modulation, IEEE Trans. on Commun., Vol. COM-29, No. 2, P.162-168, February 1981.

[4] N. Boutin, S. Morissette, and L. Dussault, Optimum Discrete Pulse Shaping in MSK-Type Signals, IEEE Trans. Commun., Vol. COM-31, No.11, P.1251-1253, November 1983.

[5] G. Rabow, Amplitude- and Time-Limited Functions With Minimum Out-of-Band Energy, IEEE Trans. Commun., Vol. COM-20, P.1150-1153, December 1972.

[6] S. A. Gronemeyer and A. L. McBride, MSK and Offset QPSK Modulation, IEEE Trans. Commun., Vol. COM-24, No.8, P.809-820, August 1976.

[7] S. Pasupathy, Minimum Shift Keying, A spectrally efficient modulation, IEEE Communications Magazine, Vol.17, P.14-22, July 1979.

[8] M. Maqusi, Applied Walsh Analysis, Wiley-Heyden, London, 1981.

[9] F. de Jager and C. B. Dekker, Tamed frequency modulation-A novel method to achieve spectrum economy in digital transmission, IEEE Trans. Commun., Vol.COM-26, No.5, P.534-542, May 1978. 